Pacific Journal of Mathematics

ON FIXED POINTS OF ZERO INDEX IN ASYMPTOTIC FIXED 


\section{ON FIXED POINTS OF ZERO INDEX \\ IN ASYMPTOTIC FIXED POINT THEORY}

\section{Christian C. Fenske and Heinz-Otto Peitgen}

The fixed point theory for mappings of compact attraction type is used to obtain some new asymptotic fixed point theorems. These investigations have been motivated by the properties of ejective and attractive fixed points. Special emphasis is given to those situations where the fixed points are of zero index. The generality of the approach admits the useful formula ind $(X, f, F)=\Lambda(f, X)-\Lambda(f, X \backslash F)$ which allows the computation of the index of $F \subset \operatorname{Fix}(f: X \rightarrow X)$ in terms of Lefschetz numbers.

In this paper we continue the study of isolated fixed points which are characterized by certain local or global asymptotic properties of the underlying mapping. Our aim is to show that isolated fixed points of this sort are always of zero index if they satisfy a certain geometric boundary condition expressed in terms of a condition on the homology groups of the underlying space. Examples and a new explicit formula for the index of such points will indicate that this geometric boundary condition seems to be the best condition to establish such index characterizations. In a particular case, i.e. if the mapping can be considered as a member of a semi-flow on a nice space $X$, we will show that the index of a certain isolated fixed point $x_{0}$ is given by the difference of the Euler characteristics of $X$ and $X \backslash\left\{x_{0}\right\}$.

In particular, our main results contain the case when the isolated fixed point is repulsive or ejective and in this sense some of the results can be considered to be extensions of work done in [6], [15], [16], [17] and [19]. Moreover, it turns out that our index characterizations can be proved without using any type of $(\bmod p)$-result as given in [18], [20], [21], [22] and [23].

The key for this improvement is simply to use the fixed point theory of a more general class of maps, which includes compact maps, and some basic point set topological constructions.

For concrete applications of the general results we are concerned with, the reader is referred to the fundamental papers of R. D. Nussbaum [14], [15] and [16].

o. Preliminaries. In what follows an essential use will be made of the notion of the Lefschetz number in the generalized sense as 
given by J. Leray [10] and the fixed point index for metric ANR's developed by A. Granas in [8].

Let $E$ be a graded vector space over the field of rational numbers, $\Phi$ an endomorphism of degree zero and $N(\Phi)=\cup_{n>0} \operatorname{ker}\left(\Phi^{n}\right)$. Then $\Phi$ is said to be a Leray endomorphism iff $\tilde{E}=E / N(\Phi)$ is of finite type. In that case we define $\operatorname{Tr}(\Phi)=\operatorname{trace}(\tilde{\Phi})$, where $\Phi: \tilde{E} \rightarrow \tilde{E}$ is the induced endomorphism. The generalized Lefschetz number of $\Phi$ is denoted by $\Lambda(\Phi)$ and is given by the formula

$$
\Lambda(\Phi)=\sum_{q}(-1)^{q} \operatorname{Tr}\left(\Phi_{q}\right)
$$

We let $H_{*}$ denote the singular homology functor with rational coefficients and $f_{*}$ is the abbreviation for $H_{*}(f)$, where $f$ is a map. A map $f: X \rightarrow X$ is said to be a Lefschetz map iff $f_{*}: H_{*}(X) \rightarrow H_{*}(X)$ is a Leray endomorphism and in that case the generalized Lefschetz number of $f$ is given by $\Lambda(f, X)=\Lambda\left(f_{*}\right)$.

For triples $(X, f, U)$, where $X$ is a metric ANR, $U$ is open in $X$ and $f: U \rightarrow X$ is a compact, admissible map (i.e. Fix $(f)=\{x \in U \mid f(x)=x\}$ is compact in $U)$, a fixed point index $i(X, f, U)$ is defined in [8] which satisfies the standard properties including the strong normalization property $i(X, f, X)=\Lambda(f, X)$.

By $\mathcal{U}\left(x_{0}\right)$ we denote the system of open neighbourhoods of a point $x_{0}$ in a space $X$.

Most of the results will be given for metric ANR's. The consideration of this class of spaces is of interest because it contains important examples both in topology and analysis; for example,

- convex subsets of a linear normed space

- open subsets of a linear normed space

- finite polyhedra

-- compact manifolds

- Banach manifolds

- open subsets of such spaces.

The class of maps - always assumed to be continuous - which we want to consider is given in the following definitions.

(0.1) Definition (cf. [7]). Let $X$ be a topological space, $f: X \rightarrow X$ a map, $A$ and $K$ two subsets of $X$.

(0.1.1) The set $o_{K}=\cup_{n} f^{n}(K)$ is said to be the orbit of $K$ under $f$ and $f^{n}(K)$ is the $n$th member of $o_{K}$.

(0.1.2) We say that $A$ absorbs $K$ provided almost all members of $0_{K}$ are contained in $A$.

(0.1.3) We say that $A$ attracts a point $x \in X$ provided the intersection $\operatorname{cl}\left(o_{x}\right) \cap A$ is not empty. 
(0.1.4) We say that $A$ is an attractor for $f$ provided it attracts all the points in $X$.

(0.1.5) We say that $f$ is of compact attraction provided

(i) $f$ has a compact attractor and

(ii) $f$ is a locally compact map.

(0.1.6) We say that $A$ is a stable, compact attractor for $f$ provided

(i) $A$ is a compact attractor for $f$ and $f(A) \subset A$ and

(ii) $A$ possesses arbitrarily small neighbourhoods $U$ such that $f(U) \subset U$ and $\operatorname{cl} f(U)$ is compact in $U$.

One encounters attractors in analysis when one considers the map of translation along trajectories of differential equations or functional differential equations which satisfy various assumptions of stability.

We are indebted to Andrzej Granas for acquainting us with the Lefschetz theory for mappings of compact attraction (cf. [7]) and the concept of a Lefschetz map for pairs of spaces which turns out to be very useful in $§ I V$.

Typical examples of isolated fixed points that we want to study are the following:

(0.2) Definition (cf. [2] and [3]). Let $X$ be a topological space, $x_{0} \in X$ and $f: X \backslash\left\{x_{0}\right\} \rightarrow X$ a map.

(0.2.1) We say that $x_{0}$ is a repulsive point of $f$ relative to $U \in \mathcal{U}\left(x_{0}\right)$ provided for any $V \in \mathcal{U}$ there is an $n_{0} \in \mathbf{N}$ such that $f^{n}(X \backslash V)$ is defined and

$$
f^{n}(X \backslash V) \subset X \backslash U
$$

whenever $n \geqq n_{0}$.

(0.2.2) We say that $x_{0}$ is an ejective point of $f$ relative to $U \in \mathcal{U}\left(x_{0}\right)$ provided for any $x \in U$ there is an $n_{x} \in \mathbf{N}$ such that $f^{n_{x}}(x)$ is defined and

$$
f^{n_{x}}(x) \in X \backslash U
$$

In view of applications (cf. [15] and [16]) we do not assume that $f$ is continuously defined at $x_{0}$. In case $f$ is continuously defined at $x_{0}$ and $f\left(x_{0}\right)=x_{0}(0.2)$ provides the definition of a repulsive (resp. ejective) fixed point of $f$.

(0.2.3) Let $f: X \rightarrow X$. We say that $x_{0}$ is an attractive fixed point of $f$ relative to $U \in \mathcal{U}\left(x_{0}\right)$ provided for any $V \in \mathcal{U}\left(x_{0}\right)$ there is an $n_{0} \in \mathbf{N}$ such that 


$$
f^{n}(U) \subset V
$$

whenever $n \geqq n_{0}$.

1. Ejective and repulsive points. Our aim in this section is to establish a relation between ejective and repulsive (fixed) points which will allow us to transfer the results about repulsive (fixed) points to ejective (fixed) points. This is of importance because in concrete applications it is, of course, much easier to verify that a point is ejective rather than repulsive. The result that we give here is partially based on a trick from [3] and is stated for Banach manifolds. It can be easily stated for other spaces (for example; compact, convex, infinite dimensional subsets of a Banach space, cones in Banach spaces) by adding or leaving out obvious assumptions.

(1.1) LEMMA. Let $X$ be a metrizable, infinite dimensional Banach manifold, $x_{0} \in X, U \in U\left(x_{0}\right)$ and $f: X \rightarrow X$ a compact map such that

(1.1.1) $x_{0}$ is an ejective fixed point of $f$ relative to $U \in U\left(x_{0}\right)$. Then there is a compact map $h: X \times[0,1] \rightarrow X$ and there is $W \in \mathcal{U}\left(x_{0}\right)$ such that

(1.1.3) for all $x \in \partial W$ and $t \in[0,1] h(x, t) \neq x$;

(1.1.4) $\quad x_{0}$ is an ejective fixed point of $g(x)=h(x, 1)$ and $f(x)=$ $h(x, 0)$;

If in addition

(1.1.5) $g\left(X \backslash\left\{x_{0}\right\}\right) \subset X \backslash\left\{x_{0}\right\}$. then

(1.1.2) for any $V \in \mathcal{U}\left(x_{0}\right)$ we have that $x_{0} \notin \operatorname{cl} f(U \backslash V)$

(1.1.6) for any $V \in \mathscr{U}\left(x_{0}\right)$ we have that $x_{0} \notin \operatorname{cl} g(X \backslash V)$.

The purpose of the lemma is to pass from a situation which is very convenient for applications via a homotopy leaving the fixed point index invariant to a situation which fits into the framework of the coming characterization results. In some sense properties (1.1.4)-(1.1.6) mean that $x_{0}$ is almost a repulsive fixed point. In particular using straightforward arguments and Lemma 1 in [3] (cf. Lemma 1.3 in [15]) one shows that actually (1.1.4)-(1.1.6) imply that $x_{0}$ is a repulsive fixed point of $g$.

If $x_{0}$ is a repulsive fixed point then (1.1.6) is obviously satisfied. On the other hand, if $x_{0}$ is only ejective even the corresponding local condition (1.1.2) need not be satisfied. This is shown by the following example which also shows that (1.1.2) can be satisfied:

(1.2) Example. Let $P$ be the cone of positive realvalued continuous functions on $[0,1]$ endowed with the sup-norm topology. Let $k:[0,1] \times[0,1] \rightarrow \mathbf{R}_{+}$be continuous and such that $0<m \leqq k(s, t) \leqq M<$ $\infty$ for all $s, t$. Then 


$$
A x(t)=\int_{0}^{1} k(s, t) x(s)^{1 / 2} d s
$$

is a completely continuous operator leaving $P$ invariant and it is easily seen that $0 \in P$ is an ejective fixed point of $A$.

(1.2.1) Now, let $\delta>0$ be arbitrary and set $x_{n}(t)=\delta t^{2 n}, n \in \mathbf{N}$; then $\left\|x_{n}\right\|=\delta A\left(x_{n}\right) \rightarrow 0$. Hence, (1.1.2) is not true for any choice of $U \in$ $\mathcal{U}(0)$.

(1.2.2) To see that (1.1.2) can be satisfied, consider the cone

$$
C=\{x \in P \mid \min x(t) \geqq m / M\|x\|\}
$$

and observe that $A$ again leaves $C$ invariant and 0 is ejective. Considered as a mapping from $C$ to $C A$ now satisfies (1.1.2).

Proof of (1.1). Let $d$ denote a metric on $X$. Choose a coordinate neighbourhood $U_{0} \in \mathcal{U}\left(x_{0}\right)$ such that $U_{0} \subset U$ and $U_{0}$ is homeomorphic to some Banach space $B$ via a homeomorphism $H$. Choose $W \in \mathcal{U}\left(x_{0}\right)$ such that $\operatorname{cl} W \subset U_{0}$ and

$$
d(X \backslash U, \operatorname{cl} W)=\delta_{W}>0 .
$$

Set $C=\overline{\operatorname{cov}}\left\{H(\operatorname{cl} f(X) \cap \mathrm{cl} W) \cup I^{\infty}\right\}$, where $I^{\infty}$ is some infinite dimensional compact subset of $B$. By a theorem of Klee [9] we find a homeomorphism $G: C \rightarrow C$ such that $G\left(H\left(x_{0}\right)\right)$ is an extreme point of $C$. Define $F: C \rightarrow X$ by $F(x)=H^{-1}\left(G^{-1}(x)\right)$, then $K=F(C)$ is a compact subset of $U_{0}, x_{0} \in K$ and $\operatorname{cl} f(X) \cap \operatorname{cl} W \subset K$.

$$
\text { Definition of } h: X \times[0,1] \rightarrow X \text {. }
$$

Define $\lambda: X \rightarrow[0,1)$ by $\lambda(x)=1-1 /(1+\min \{d(x, \operatorname{cl} W), d(f(x)$, $X \backslash W)\})$. Then $\lambda$ is continuous and has the following properties:

$$
\begin{array}{ll}
\lambda(x)=0 & \text { iff } x \in \operatorname{cl} W \text { or } f(x) \in X \backslash W \\
\lambda(x) \neq 0 & \text { iff } x \notin \operatorname{cl} W \text { and } f(x) \in W .
\end{array}
$$

Now choose $z \in C, z \neq F^{-1}\left(x_{0}\right)$, and define

$$
h(x, t)= \begin{cases}F\left\{(1-t \lambda(x)) F^{-1}(f(x))+t \lambda(x) z\right\}, & \text { if } \lambda(x) \neq 0 \\ f(x), & \text { if } \lambda(x)=0 .\end{cases}
$$

Observe that $h$ is well defined since $\lambda(x) \neq 0$ implies $f(x) \in W$, i.e. $f(x) \in K$. Moreover, observe that $h$ is continuous, compact and 
$h(x, 0)=f(x) . \quad(1.1 .3)$ follows from the fact that $x_{0}$ is an ejective fixed point of $f$ relative to $U$ and $h(x, t)=f(x)$ for all $x \in \partial W \subset U$.

The proof of (1.1.4) is obvious because $g$ coincides with $f$ in $W$.

Proof of (1.1.5). Assume there is $x \neq x_{0}$ such that $g(x)=x_{0}$. Then two cases might occur:

(a) $\lambda(x)=0$; hence $x \in \mathrm{cl} W$ - which is impossible, since $x_{0}$ is ejective, or $g(x)=f(x) \in X \backslash W-$ which is impossible, since $g(x)=$ $f(x)=x_{0} \in W$.

(b) $0<\lambda(x)<1$; then $F^{-1}\left(x_{0}\right)=(1-\lambda(x)) F^{-1}(f(x))+\lambda(x) z$, and again we arrive at a contradiction, since $F^{-1}\left(x_{0}\right)$ is an extreme point, but $z \neq F^{-1}\left(x_{0}\right)$.

To prove (1.1.6) we need the following:

(1.1.10) If $\left(x_{n}\right)$ is a sequence in $X$ such that $g\left(x_{n}\right) \rightarrow x_{0}$ then $x_{0}$ is an accumulation point of $\left(f\left(x_{n}\right)\right)$.

Proof of (1.1.10). Since $\lambda\left(x_{n}\right) \in[0,1)$ we may choose a subsequence $\left(x_{n}\right)$ such that $\lambda\left(x_{n}\right) \rightarrow \lambda_{0} \in[0,1]$. We will show that $\lambda_{0}=0$. Assume $\lambda_{0}=1$; then $\left(1-\lambda\left(x_{n}\right)\right) F^{-1}\left(f\left(x_{n}\right)\right) \rightarrow 0 \in B$, since $\left(F^{-1}\left(f\left(x_{n}\right)\right)\right) \subset C$ is bounded. Hence, $g\left(x_{n}\right) \rightarrow F(z)$ and $g\left(x_{n}\right) \rightarrow x_{0}$; this is a contradiction. Now $\lambda_{0}<1$ implies that $1 /\left(1-\lambda\left(x_{n}\right)\right)$ is a convergent sequence. Hence, $F^{-1}\left(f\left(x_{n}\right)\right)=\left(F^{-1}\left(g\left(x_{n}\right)\right)-\lambda\left(x_{n}\right) z\right) /\left(1-\lambda\left(x_{n}\right)\right)$ is a convergent sequence in $C$ and $F^{-1}\left(f\left(x_{n}\right) \rightarrow u=\left(F^{-1}\left(x_{0}\right)-\lambda_{0} z\right) /\left(1-\lambda_{0}\right) \in\right.$ $C$. Of course, we have $u \neq z$ because $u=z$ would imply $F^{-1}\left(x_{0}\right)=$ $z$. Assume $\lambda_{0} \neq 0$; then

$$
\begin{array}{cc}
F^{-1}\left(g\left(x_{n}\right)\right) & \left(1-\lambda\left(x_{n}\right)\right) F^{-1}\left(f\left(x_{n}\right)\right)+\lambda\left(x_{n}\right) z \\
F^{-1}\left(x_{0}\right) & \left(1-\lambda_{0}\right) u+\lambda_{0} z
\end{array}
$$

and again we have a contradiction to the extremality of $F^{-1}\left(x_{0}\right)$. However, $\lambda_{0}=0$ implies $F^{-1}\left(f\left(x_{n}\right)\right) \rightarrow F^{-1}\left(x_{0}\right)$, i.e. $f\left(x_{n}\right) \rightarrow x_{0}$.

Proof of (1.1.6). Assume that there is $V_{0} \in U\left(x_{0}\right)$ such that $x_{0} \in$ $\operatorname{cl} g\left(X \backslash V_{0}\right)$, i.e. there is $\left(x_{n}\right) \subset X \backslash V_{0}$ such that $g\left(x_{n}\right) \rightarrow x_{0}$. If infinitely many $x_{n}$ were in $U \backslash V_{0}$ we would have $g\left(x_{n}\right) \rightarrow x_{0}$ and from (1.1.10) $f\left(x_{n_{t}}\right) \rightarrow x_{0}$ for a subsequence in $U \backslash V_{0}$, which would be a contradiction to (1.1.2). Thus, we may assume that $\left(x_{n}\right) \subset X \backslash U$. But $\left(x_{n}\right) \subset X \backslash U$ implies $d\left(x_{n}, \mathrm{cl} W\right) \geqq \delta_{W}>0$ for all $n$, and $f\left(x_{n_{t}}\right) \rightarrow x_{0}$ implies $d\left(f\left(x_{n_{i}}\right), X \backslash W\right) \geqq$ $\delta_{0}>0$ for almost all $n_{t}$. Hence, from the definition of $\lambda$, we have that

$$
\lambda\left(x_{n_{i}}\right) \geqq \lambda_{0}\left(\delta_{W}, \delta_{0}\right)>0 \text { for almost all } n_{t},
$$

and this again leads to a contradiction to the extremality of $F^{-1}\left(x_{0}\right)$. 


\section{(1.3) REMARKS.}

(1.3.1) Let $X$ be a subset of a linear, normed space, $x_{0} \in X$, $f: X \rightarrow X$ completely continuous, $U \in \mathcal{U}\left(x_{0}\right)$ and $K \subset X$ a closed subset such that (1.1.1) and (1.1.2) are satisfied. Assume that $\operatorname{cl} U \cap \operatorname{cl} f(X) \subset K$ and $K$ is homeomorphic to a closed, convex subset of a linear, normed space such that $x_{0}$ corresponds to an extreme point. Then the conclusions of (1.1) are true with almost no change of the proof, except that in this case the homotopy is completely continuous.

(1.3.2) Applying (1.3.1) to the situation where $X$ is a cone in a Banach space and $x_{0}=0$, i.e. $x_{0}$ is an extreme point, one gets a strengthening of a result of Nussbaum [15].

(1.3.3) Obvious modifications of the formulation of (1.1), (1.3.1) and (1.3.2) and their proofs give immediately corresponding results for the case when $f: X \backslash\left\{x_{0}\right\} \rightarrow X$ and $x_{0}$ is only an ejective point.

II. Mappings of compact attraction. The following purely point set topological Lemma (2.1) was communicated to us by A. Granas. It also follows by a combination of Lemma 5.1 in [7] and an argument used in Theorem 2.1 in [7]. The construction used there is attributed to unpublished ideas of Eells and Gleason-Palais and has been used in similar situations in [4], [5], [6], [12], [13] and [19].

(2.1) Lemma. Let $X$ be a metric space, $f: X \rightarrow X$ a mapping of compact attraction and $A$ a compact attractor for $f$. Then there is an open neighbourhood $U$ of $A$ such that

(2.1.1) $f(U) \subset U$ and

(2.1.2) $f_{i U}: U \rightarrow U$ is a compact map.

G. Fournier in [7] uses this fact to extend the notion of a Lefschetz number to mappings of compact attraction. In fact, he shows that $\Lambda(f, X)$ is defined and $\Lambda(f, X)=\Lambda\left(f_{\mid U}, U\right)$ if $X$ is a metric ANR.

(2.2) Corollary. Let $X$ be a metric space, $f: X \rightarrow X$ a mapping of compact attraction and $A$ a compact attractor of $f$.

(2.2.1) Then there is a stable, compact attractor $A_{0} \supset A$ for $f$.

Proof. Set $A_{0}=A \cup \operatorname{cl} f(U)$, where $U$ is a neighbourhood of $A$ satisfying (2.1.1) and (2.1.2). Then $f\left(A_{0}\right) \subset f(A \cup \operatorname{cl} f(U)) \subset A_{0}$. Clearly, if $V$ is a neighbourhood of $A_{0}$ and $V \subset U$ we have $\operatorname{cl} f(V) \subset \operatorname{cl} f(U) \subset$ $A_{0} \subset V$.

Corollary (2.2) shows that our concept coincides with Nussbaum's concept of an attractor (cf. [13]). 
(2.3) Lemma. Let $X$ be a metric space, $f: X \rightarrow X$ a locally compact map and $A \subset X$ an invariant compact set. Assume that each open neighbourhood of $A$ absorbs compact sets in $X$. Then there exist arbitrarily small open neighbourhoods $U$ of $A$ such that

(2.3.1) $f(U) \subset U$ and

(2.3.2) $f_{\mid U}: U \rightarrow U$ is a compact map.

Proof. Even if $A$ is a compact, invariant attractor for $f$ the conclusion of (2.3) does not follow from (2.2), because, in general, one has to choose there a bigger invariant attractor $A_{0}$. By Lemma 5 in [13] there is $W \in \mathcal{U}(A)$ arbitrarily small such that $f(W) \subset W$. Since $f$ is locally compact we may assume that $\operatorname{cl} f(W)$ is compact. Now $W$ absorbs points in $X$, hence compact sets in $X$, and we find $m_{0} \in \mathbf{N}$ such that $\quad \operatorname{cl} f^{m}(W) \subset \operatorname{cl} f^{m-1}(\operatorname{cl} f(W)) \subset W$, whenever $m \geqq m_{0}$. Set $C=$ $A \cup \mathrm{cl} f^{m_{0}}(W)$ and observe that $f(C) \subset C$ and $C \subset W$ and $f^{-m_{0}}(C) \supset$ $W$. Choose $W_{1}, \cdots, W_{m_{0}-1} \in \mathcal{U}(C)$ such that

$$
C \subset W_{m_{0-1}} \subset \mathrm{cl} W_{m 0-1} \subset \cdots W_{1} \subset \operatorname{cl} W_{1} \subset W
$$

and set

$$
U=W \cap \bigcap_{j=1}^{m_{0}-1} f^{-j}\left(W_{\jmath}\right)
$$

Clearly, $C \subset U \subset W$ and one shows easily that $\operatorname{cl} f(U) \subset U$.

The next lemma, again purely point set topological, is motivated by Theorem (3.1) in [17] and will enable us to prove an extension and generalization of this theorem (see §IV).

(2.4) Lemma. Let $X$ be a metric space, $f: X \rightarrow X$ a map of compact attraction and $V$ open in $X$ such that

(2.4.1) there is an $n_{0}$ such that $f^{n}(X \backslash V) \subset X \backslash \mathrm{cl} V$, whenever $n \geqq n_{0}$. Then there is $Y$ open in $X \backslash \mathrm{cl} V$ such that

(2.4.2) $f(Y) \subset Y, f_{Y}: Y \rightarrow Y$ is a compact map and

(2.4.3) $Y$ absorbs compact sets in $X \backslash V$.

Proof. If $A$ is any attractor of $f$, then Lemma (2.1) implies that there exists $U \in U(A)$ such that $\operatorname{cl} f(U)$ is compact in $U$. Hence, $A_{0}=A \cup \mathrm{cl} f(U)$ is a compact invariant attractor. Note, that $U$ absorbs points in $X$, and, hence, compact subsets of $X$. Thus, if $K \subset X$ is a compact set there is an $m_{0} \in \mathbf{N}$ such that $f^{m}(K) \subset U$, whenever $m \geqq$ $m_{0}$. Then $f^{m+1}(K) \subset f(U) \subset A \cup \operatorname{cl} f(U)$. Hence, $A_{0}$ absorbs compact sets in $X$. Set $B=f^{n_{0}}((A \cup \operatorname{cl} f(U)) \cap X \backslash V)$ and observe that $B$ is a compact subset of $X \mid \mathrm{cl} V$. Set $C=\bigcup_{j=0}^{n_{0}-1} f^{j}(B)$ and observe that $C$ is in $X \backslash \mathrm{cl} V$ and $f(C) \subset C$. 
Moreover, if $K$ is any compact set in $X \backslash V$, we have that $f^{m_{0}}(K) \subset A \cup$ $\operatorname{cl} f(U)$ for some $m_{0} \in \mathbf{N}$; thus $f^{m_{0}+2 m_{0}}(K) \subset B \subset C$, i.e. $C$ absorbs compact sets in $X \backslash V$. Since $f$ is locally compact, we find $W \in \mathcal{U}(C)$ such that $\operatorname{cl} W \subset X \backslash \mathrm{cl} V$ and $\operatorname{cl} f(W)$ is a compact subset of $X \backslash V$. Hence, there is $k_{0} \in \mathbf{N}$ such that $\mathrm{cl} f^{k_{0}}(W) \subset C$. Now choose as in the proof of (2.3) $W_{1}, \cdots, W_{k_{0}-1} \in U(C)$ and construct an open set $Y$ with the properties (2.4.2) and (2.4.3).

(2.5) Corollary. Let $X$ be a metric space, $f: X \rightarrow X$ a map of compact attraction, $x_{0} \in X$ a repulsive fixed point of $f$ relative to $U \in \mathcal{U}\left(x_{0}\right)$ and $V$ any neighbourhood of $x_{0}$ such that $\operatorname{cl} V \subset U$. Then $f: X \backslash\left\{x_{0}\right\} \rightarrow X \backslash\left\{x_{0}\right\}$ has a stable, compact attractor $A \subset X \backslash \mathrm{cl} V$.

Proof. Choose $V \in \mathcal{U}\left(x_{0}\right), \mathrm{cl} V \subset U$. Then there is an $n_{0} \in \mathbf{N}$ such that $f^{n}(X \backslash V) \subset X \backslash U \subset X \backslash \mathrm{cl} V$, whenenver $n \geqq n_{0}$. By Lemma (2.4) we find $Y \subset X \mid \mathrm{cl} V$ such that $A=\operatorname{cl} f(Y)$ is a compact, invariant subset of $Y$ which absorbs compact sets of $X \backslash V$. If $x \neq x_{0}$ then $f^{m}(x) \in X \backslash U$ for some $m \in \mathbf{N}$; hence, $A$ absorbs points in $X \backslash\left\{x_{0}\right\}$.

If $x_{0}$ is only an ejective fixed point and if we assume that $f\left(X \backslash\left\{x_{0}\right\}\right) \subset X \backslash\left\{x_{0}\right\}$ - which is reasonable by Lemma (1.1) - an only slightly less sharp result is possible.

(2.6) Lemma. Let $X$ be a metric space, $f: X \rightarrow X$ a map of compact attraction, $x_{0} \in X$ an ejective fixed point of $f$ relative to $U \in U\left(x_{0}\right)$ and $f\left(X \backslash\left\{x_{0}\right\}\right) \subset X \backslash\left\{x_{0}\right\}$. Then $f: X \backslash\left\{x_{0}\right\} \rightarrow X \backslash\left\{x_{0}\right\}$ has a stable, compact attractor.

Proof. By Corollary (2.2) we find a stable, compact attractor $A$ for $f: X \rightarrow X$ which absorbs points of $X$. Set $B=A \cap X \backslash U$ and observe that $B$ attracts points of $X \backslash\left\{x_{0}\right\}$.

The close connection between the notion of ejectivity and the notion of a compact attractor is shown by the

(2.7) Lemma. Let $X$ be a metric space, $f: X \rightarrow X$ a map of compact attraction and $F \subset X$ a closed subset such that $f(X \backslash F) \subset X \backslash F$. Then the following are equivalent

(2.7.1) $f_{\mid X \backslash F}: X \backslash F \rightarrow X \backslash F$ is a map of compact attraction;

(2.7.2) $F$ is an ejective set for $f$.

Proof. Lemma (2.6) indicates one direction of the proof. By Corollary (2.2) we find a stable, compact attractor $A \subset X \backslash F$ which absorbs compact sets in $X \backslash F$. Choose $V \in \mathcal{U}(F)$ such that $\operatorname{cl} V \cap A=$ 
$\varnothing$. Let $x$ be in $V \backslash F$. Then $f^{n}(x) \in A$ for some $n \in \mathbf{N}$. Hence, $f^{n}(x) \notin V$, i.e. $F$ is an ejective set for $f$ relative to $V$.

\section{The fixed point index for mappings of compact}

attraction. The following definition is based on the definition of a fixed point index for compact maps of metric ANR's as given by A. Granas in [8] and his generalization to admissible maps $f: U \rightarrow X(X$ is a metric ANR, $U$ is open in $X$ and Fix $(f)$ is compact in $U$ ) such that for some neighbourhood $V$ of $\operatorname{Fix}(f)$ the restriction $f_{\mid V}$ is a compact map. Note that if $f: U \rightarrow X$ is an admissible and locally compact map then there is $V \in \mathcal{U}(\operatorname{Fix}(f))$ such that $f_{\mid V}$ is a compact map.

(3.1) Definition. Let $X$ be a metric ANR, $f: X \rightarrow X$ a map of compact attraction and $U$ open in $X$ such that $f(x) \neq x$ for all $x \in$ $\partial U$. Choose $Y$ open in $X$ such that $Y$ absorbs compact sets in $X$, $f(Y) \subset Y$ and $f_{\mid Y}: Y \rightarrow Y$ is a compact map. Define

$$
i(X, f, U)=i(Y, f, Y \cap U) .
$$

From $([8], \S 10)$ we know that $i(Y, f, Y \cap U)$ is well defined, since $Y$ is itself an ANR. The existence of such an open set $Y$ is guaranteed by Lemma (2.1). An easy computation shows that this definition is independent of the choice of $Y$. Moreover, this index is a fixed point index in the usual sense, i.e. it satisfies the following axioms:

(3.2) Additivity. Let $U, U_{1}, U_{2}$ be open in $X$ such that $U_{1}, U_{2} \subset$ $U, U_{1} \cap U_{2}=\varnothing$ and $f(x) \neq x$ for all $x \in \operatorname{cl} U \backslash\left(U_{1} \cup U_{2}\right)$. Then

$$
i(X, f, U)=i\left(X, f, U_{1}\right)+i\left(X, f, U_{2}\right) .
$$

(3.3) Номотору. Let $h: X \times[0,1] \rightarrow X$ be a map such that for all $x \in \partial U$ and for all $t \in[0,1] h(x, t) \neq x$. Let $H: X \times[0,1] \rightarrow X \times[0,1]$ be defined by $H(x, t)=(h(x, t), t)$. Assume that $H$ is a map of compact attraction, then

$$
i\left(X, h_{0}, U\right)=i\left(X, h_{1}, U\right), \text { where } h_{t}(x)=h(x, t) .
$$

(3.4) Commutativity. Let $X, Z$ be metric ANR's, $f: X \rightarrow Z$ and $g: Z \rightarrow X$ locally compact maps, $U$ open in $X$ and $V$ open in $Z$ such that

(3.4.1) $f(U) \subset V$ and

(3.4.2) for all $x \in \partial U g f(x) \neq x$ (which is equivalent to: for all $\left.y \in \partial g^{-1}(U) f g(y) \neq y\right)$. Then, if

(3.4.3) $f g$ has a compact attractor then $g f$ has a compact attractor and, in either case, 


$$
i(X, g f, U)=i\left(Z, f g, g^{-1}(U)\right) .
$$

Proof of (3.4). Let $A$ be an attractor for $g f$. Then $f(A)$ is an attractor for $f g$. Choose $Y$ open in $X$ such that $g f(Y) \subset Y$ and $(g f)_{\mid Y} ; Y \rightarrow Y$ is a compact map and absorbs compact sets in $X$. For any $x \in \operatorname{cl} g f(Y)$ choose $U_{x} \subset Y$ such that $\operatorname{cl} f\left(U_{x}\right)$ is compact. The compactness of $g f(Y)$ implies that

$$
\tilde{Y}=\bigcup_{j=1}^{n} U_{x_{j}} \supset \operatorname{clg} g(Y) \subset Y .
$$

Now, $\operatorname{cl} g f(\tilde{Y}) \subset \operatorname{cl} g f(Y) \subset \tilde{Y}$ and $\tilde{Y}$ absorbs compact sets in $X$. Set $W=g^{-1}(\tilde{Y})$. Then, $\operatorname{cl} g f(\tilde{Y}) \subset \tilde{Y}$ implies that $g(\operatorname{cl} f(\tilde{Y})) \subset \tilde{Y}$ and this implies that $\operatorname{cl} f(\tilde{Y}) \subset g^{-1}(\tilde{Y})=W$. Thus,

$$
\operatorname{cl} f g(W)=\operatorname{cl} f g g^{-1}(\tilde{Y})=\operatorname{cl} f(\tilde{Y}) \subset W .
$$

Moreover, $W$ absorbs compact sets in $Z$ with respect to $f g$. To see this let $K$ be a compact set in $Z$. Then $(g f)^{n}(g K) \subset \tilde{Y}$ for some $n$. Hence,

$$
(f g)^{n+1}(K)=f(g f)^{n} g(K) \subset f(\tilde{Y}) \subset W .
$$

Finally,

$$
\begin{aligned}
i(X, g f, U) & =i(\tilde{Y}, g f, \tilde{Y} \cap U)=i\left(W, f g, g^{-1}(U \cap \tilde{Y})\right) \\
& =i\left(W, f g, g^{-1}(U) \cap W\right)=i\left(Z, f g, g^{-1}(U)\right) .
\end{aligned}
$$

(3.5) Normalization. Let $X$ be a metric ANR and $f: X \rightarrow X$ a map of compact attraction. Then

$$
i(X, f, X)=\Lambda(f, X)
$$

Proof of (3.5). Choose $Y$ open in $X$ such that (3.1.1)-(3.1.3) are satisfied. Then $i(X, f, X)=i(Y, f, Y)$. On the other hand $G$. Fournier in [7] has shown that the Lefschetz number of $f: X \rightarrow X$ is defined and $\Lambda(f, X)=\Lambda(f, Y)$ (cf. the proof of (4.8)).

IV. Some new asymptotic fixed point theorems. To prove our main results we need the following two lemmata which were communicated to us by A. Granas (cf. [1], [5] and [11]). The first is a generalization of the well known formula for the relative Euler characteristic. 
(4.1) Lemma. Let $(X, A)$ be a pair of topological spaces and $f:(X, A) \rightarrow(X, A)$ a map. If any two among $f, f_{\mid X}: X \rightarrow X, f_{i A}: A \rightarrow A$ are Lefschetz maps, then so is the third and in that case we have

$$
\Lambda(f,(X, A))=\Lambda\left(f_{\mid X}, X\right)-\Lambda\left(f_{\mid A}, A\right) .
$$

The second lemma follows immediately from the definition of a Leray endomorphism.

(4.2) LEMMA. Let $E$ be a vector space (over the rational numbers) and $T: E \rightarrow E$ weakly-nilpotent (i.e. for all $e \in E$ there is an $n \in \mathbf{N}$ such that $\left.T^{n}(e)=0\right)$.

Then $\operatorname{Tr}(T)=0(\operatorname{Tr}(T)$ denotes the generalized trace in the sense of Leray [10]).

By the generality of our approach the following lemmata are now almost trivial, though their interpretation (cf. Lemma (2.7)) in the instance of ejective (fixed) points extends and refines the results known for such points (cf. [2], [3], [6], [15], [16], [17] and [19]).

(4.3) Lemma. Let $X$ be a metric ANR and $f: X \rightarrow X$ a map of compact attraction. Let $F \subset \operatorname{Fix}(f)$ be open and closed in Fix $(f)$. Assume that $f(X \backslash F) \subset X \backslash F$ and that $f_{\mid X \backslash F}$ is a map of compact attraction. Then

$$
i(X, f, W)=\Lambda(f, X)-\Lambda\left(f_{\mid X \backslash F}, X \backslash F\right)
$$

for any neighbourhood $W$ of $F$ such that $\mathrm{cl} W \cap \operatorname{Fix}(f) \backslash F=\varnothing$.

Proof. Since $F$ is an isolated component of Fix $(f)$ it is also a closed subset of $X$; hence, $X \backslash F$ is a metric ANR. Choose $W \in U(F)$ such that cl $W \cap \operatorname{Fix}(f) \backslash F=\varnothing$. If $A$ is any stable, compact attractor (cf. Corollary (2.2)) for $f_{\mid X \backslash F}$ and $Y$ is open in $X \backslash F$ such that $A \subset Y$ and $\operatorname{cl} f(Y)$ is compact in $Y$, then $\Lambda\left(f_{|X| F}, X \backslash F\right)=\Lambda\left(f_{\mid Y}, Y\right)$. Now, the normalization, commutativity and additivity properties of the index imply

$$
\begin{gathered}
\Lambda\left(f_{\mid Y}, Y\right)=i(Y, f, Y)=i(X, f, Y)=i(X, f, X \mid \mathrm{cl} W) \text { and } \\
i(X, f, W)=i(X, f, X)-i(X, f, X \mid \mathrm{cl} W)=\Lambda(f, X)-\Lambda\left(f_{|X| F}, X \backslash F\right) .
\end{gathered}
$$

(4.4) Corollary. Let $X$ be a metric ANR, $f: X \rightarrow X$ a map of compact attraction and $x_{0} \in X$ an ejective fixed point of $f$ relative to $W \in \mathcal{U}\left(x_{0}\right)$ such that $f(x) \neq x$ for all $x \in \partial W$ and $f\left(X \backslash\left\{x_{0}\right\}\right) \subset$ $X \backslash\left\{x_{0}\right\}$. Then 


$$
i(X, f, W)=\Lambda(f, X)-\Lambda\left(f_{|X|\left\{x_{0}\right\}}, X \backslash\left\{x_{0}\right\}\right)
$$

Note that if $f$ is already a compact map then $f_{\{X \mid\{x\}\}}$ is not a compact map. This demonstrates that the class of maps which are of compact attraction, not only leads to more general results but also, and, this seems to be more important, leads to sharper results.

Some concrete applications of (4.4) should be mentioned.

(4.5) Corollaries (cf. [2], [3], [6], [15], [16], [17] and [19]).

(4.5.1) Let $X$ be a cone in a Banach space and $f: X \rightarrow X$ a completely continuous map. Assume $x_{0}=0$ is an ejective fixed point relative to a bounded $W \in \mathcal{U}(0)$ and $f(x) \neq x$ for all $x \in \partial W$. Then

$$
i(X, f, W)=0 .
$$

(4.5.2) Let $X$ be a compact, convex, infinite dimensional subset of a Banach space and $f: X \rightarrow X$ a continuous map. Then $f$ has a nonejective fixed point.

(4.5.3) Let $X$ be a compact manifold with boundary $\partial X, f: X \rightarrow X a$ continuous map, $x_{0} \in \partial X$ an ejective fixed point of $f$ relative to $W \in \mathcal{U}\left(x_{0}\right)$ and $f(x) \neq x$ for all $x \in \partial W$. Assume that $f\left(X \backslash\left\{x_{0}\right\}\right) \subset X \backslash\left\{x_{0}\right\}$. Then

$$
i(X, f, W)=0 .
$$

(4.5.4) Let $X$ be an infinite dimensional, metrizable Banach manifold, $f: X \rightarrow X$ a compact map, $x_{0} \in X$ an ejective fixed point of $f$ relative to $W \in U\left(x_{0}\right)$ and $f(x) \neq x$ for all $x \in \partial W$.

$$
i(X, f, W)=0 .
$$

Proof of (4.5.1). By Lemma (1.1) and Remark (1.3) we may assume that $f(X \backslash\{0\}) \subset X \backslash\{0\}$. Choose $R>0$, such that $\operatorname{cl} W \cup \operatorname{cl} f(W) \subset B_{R}$, where $B_{R}=\{x \in X \mid\|x\| \leqq R\}$. Let $\rho: X \rightarrow B_{R}$ be the radial retraction and define $F: X \rightarrow X$ by $F(x)=f(\rho(x))$. Then $F$ is a compact map and $F(x) \neq 0$ for all $x \neq 0$ and 0 is an ejective fixed point of $F$ relative to $W$. Observe that $X$ and $X \backslash\{0\}$ are acyclic, thus

$$
i(X, f, W)=i(X, F, W)=\Lambda(F, X)-\Lambda\left(F_{\mid X \backslash\{0\}}, X \backslash\{0\}\right)=0 .
$$

Proof of (4.5.2). The compactness of $X$ implies that $f$ has at most finitely many ejective fixed points. By a result of Klee [9] we may assume that the ejective fixed points of $f$ are extreme points of $X$. If $x_{0}$ is a typical one we obtain $i\left(X, f, x_{0}\right)=0$ again because $X \backslash\left\{x_{0}\right\}$ is 
convex and by (1.3.1) we may assume that $f\left(X \backslash\left\{x_{0}\right\}\right) \subset X \backslash\left\{x_{0}\right\}$. Then the conclusion follows from the additivity and normalization properties of the index.

Proof of (4.5.3). Cf. remarks in [17] and [19].

Proof of (4.5.4). By Lemma (1.1) we may assume that $f\left(X \backslash\left\{x_{0}\right\}\right) \subset$ $X \backslash\left\{x_{0}\right\}$. Since $x_{0}$ is homotopy negligible, we have that

$$
\Lambda(f, X)=\Lambda\left(f_{*}\right)=\Lambda\left(\left(f_{|X|\left\{x_{0}\right\}}\right)_{*}\right)=\Lambda\left(f_{|X|\left\{x_{0}\right\}}, X \backslash\left\{x_{0}\right\}\right) .
$$

Another lemma can be stated now which is related to the characterization of ejective points (i.e. the map might not be defined in the critical point).

(4.6) Lemma. Let $X$ be a metric ANR, $F \subset X$ a closed subset, $f: X \backslash F \rightarrow X$ locally compact, $f(X \backslash F) \subset X \backslash F$ and $f_{\mid X \backslash F}$ a map of compact attraction. Then

$$
i(X, f, X \backslash \mathrm{cl} W)=\Lambda\left(f_{\mid X \backslash F^{\prime}} X \backslash F\right)
$$

for any $W$ such that $\mathrm{cl} W \cap \operatorname{Fix}(f)=\varnothing$.

Proof. See proof of (4.3).

(4.7) Corollary. Let $X$ be a metric ANR, $x_{0} \in X, f: X \backslash\left\{x_{0}\right\} \rightarrow X$ a compact map, $f\left(X \backslash\left\{x_{0}\right\}\right) \subset X \backslash\left\{x_{0}\right\}$ and $x_{0}$ an ejective point of $f$ relative to $W \in U\left(x_{0}\right)$ and $f(x) \neq x$ for all $x \in \partial W$. Then

$$
i(X, f, X \backslash \mathrm{cl} W)=\Lambda\left(f_{\{X \backslash\{0,0\}}, X \backslash\left\{x_{0}\right\}\right) .
$$

Proof. We have that $\operatorname{cl} f\left(X \backslash\left\{x_{0}\right\}\right) \cap X \backslash W$ is a compact attractor for $f_{X\{\langle x,\}} \cdot$

(4.7) is an extension of Nussbaum's result in [15]. In the previous results (4.3) and (4.4) we certainly obtain fixed points of zero index if $j: X \backslash F \rightarrow X$ induces isomorphisms in $H_{*}$ and (4.5) provides simple examples for this situation. The following results are devoted to fixed points of zero index.

(4.8) Theorem. Let $X$ be a metric ANR and $f: X \rightarrow X$ a map of compact attraction. Let $F \subset$ Fix $(f)$ be open and closed in Fix $(f)$. Assume that $f(X \backslash F) \subset X \backslash F$ and that $f_{\mid X \backslash F}$ is a map of compact attraction. Let $A \subset X \backslash F$ be a stable, compact attractor for $f_{|X| F}$. If 
(4.8.1) $j: X \backslash F \rightarrow X$ induces isomorphisms in $H_{*}$; or

(4.8.2) there is an open neighbourhood $U$ of $F$ such that $\operatorname{cl} U \cap A=$ $\varnothing$ and $j: X \backslash U \rightarrow X$ induces isomorphisms in $H_{*}$; or

(4.8.3) there is an open neighbourhood $V$ of $A$ such that $\operatorname{cl} V \cap F=$ $\varnothing$ and $j: V \rightarrow X$ induces isomorphisms in $H_{*}$; then

(4.8.4) $i(X, f, W)=0$ for any open neighbourhood $W$ of $F$ such that $\operatorname{cl} W \cap \operatorname{Fix}(f) \backslash F=\varnothing$ and

\section{(4.8.5) $\Lambda(f, X) \neq 0$ implies that $F \neq \operatorname{Fix}(f)$.}

Proof. Since $F$ is an isolated component of Fix $(f)$ it is a closed subset of $X$, hence, $X \backslash F$ is a metric ANR. The proof of (4.8.1) follows by the same argument as (4.5.4).

Proof of (4.8.2). Since $A$ is a stable, compact attractor in $X \backslash F$ we can find $Y$ open in $X \backslash F$ such that $\operatorname{cl} Y \cap \operatorname{cl} U=\varnothing, \operatorname{cl} f(Y)$ is compact in $Y$ and $Y$ absorbs compact sets in $X \backslash F$. It suffices to prove $\Lambda(f, X)=$ $\Lambda\left(f_{Y}, Y\right)$ because the additivity, commutativity and normalization properties of the index imply

$$
\begin{aligned}
\Lambda(f, X)=i(X, f, U)+i(X, f, Y) & =i(X, f, U)+i(Y, f, Y) \\
& =i(X, f, U)+\Lambda\left(f_{\mid Y}, Y\right)
\end{aligned}
$$

By Lemmata (4.1) and (4.2) we obtain $\Lambda(f, X)=\Lambda\left(f_{\mid Y}, Y\right)$ after we have proved that $\tilde{f}_{*}: H_{*}(X, Y) \rightarrow H_{*}(X, Y), \tilde{f}(x)=f(x)$, is weaklynilpotent. Hence, we have to show that for any $a \in H_{*}(X, Y)$ there is an $n \in \mathbf{N}$ such that $\tilde{f}_{*}^{n}(a)=0$. Observe that assumption (4.8.2), together with the five lemma, imply that the inclusion $k:(X \backslash U, Y) \rightarrow(X, Y)$ induces isomorphisms in $H_{*}$. Let $a$ be in $H_{*}(X, Y)$; then we find $b \in H_{*}(X \backslash U, Y)$ such that $a=k_{*} b$. Since $H_{*}$ has compact supports, we find a compact pair $(K, L), L \subset K \subset X \backslash U$ and $L \subset Y$, such that $b=i_{L}^{K}(c)$, where $c \in H_{*}(K, L)$ and $i_{L}^{K}:(K, L) \rightarrow(X \backslash U, Y)$ denotes the inclusion. Now, $Y$ absorbs compact sets of $X \backslash U \subset X \backslash F$; hence, there is an $n_{K}$ such that $f^{n_{K}}(K) \subset Y$. Denote $\left(\tilde{f}^{n_{K}}\right)_{\mid(K, L)}$ by $g_{K}$. Then the following diagram of pairs is commutative

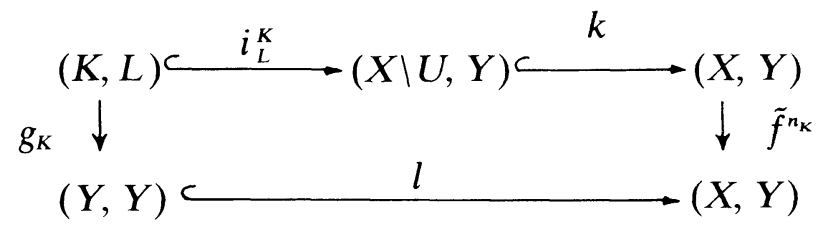


Thus, $\left(\tilde{f}_{*}\right)^{n_{K}} \circ k_{*} \circ i_{L}^{K}(c)=l_{*} \circ g_{K^{*}}(c)=0$; which implies $\left(\tilde{f}_{*}\right)^{n_{K}}(a)=0$.

Proof of (4.8.3). We find $Y$ open in $X \backslash F$ such that $A \subset Y \subset V$ and $\operatorname{cl} f(Y)$ is compact in $Y$ and $Y$ absorbs compact sets in $X \backslash F$. Now, we can follow the arguments of the proof of (4.8.2) replacing $X \backslash U$ by $V$.

Note that by Lemma (2.7) we can consider $F$ as a repulsive or ejective fixed point.

In ([17], Theorem 3.1) the second author gave an application of what has recently come to be known as the $(\bmod p)$-theorem of Zabre ̌̌ko-Krasnosel'skiǐ [23] and Steinlein [20], [21] and [22]. The next theorem is a strengthening and generalization of the corresponding result in [17] and we will suggest a proof without using any type of $(\bmod p)$ argument. Moreover, this result has a very useful interpretation in cones of Banach spaces (i.e. asymptotic expansion and asymptotic compression of a cone cf. [14]) which will be carried through in another paper.

(4.9) Theorem. Let $X$ be a metric ANR, $f: X \rightarrow X$ a map of compact attraction, $U$ open in $X$ such that

(4.9.1) there is an $n_{0} \in \mathbf{N}$ such that $f^{n}(X \backslash U) \subset X \backslash \mathrm{cl} U$, whenever $n \geqq n_{0}$, and $j: X \backslash U \rightarrow X$ induces isomorphisms in $H_{*}$. Then

$$
i(X, f, U)=0 \text { and } i(X, f, X \mid c l U)=\Lambda(f, X) .
$$

Proof. By Lemma (2.4) we find $Y$ open in $X \backslash \operatorname{cl} U$ such that $\operatorname{cl} f(Y)$ is compact in $Y$ and $Y$ absorbs compact sets in $X \backslash U$. Following the proof of (4.8.2) we obtain $\Lambda(f, X)=\Lambda\left(f_{\mid Y}, Y\right)$ which implies the conclusion by the same arguments as before.

Clearly, Theorem (4.9) especially characterizes repulsive fixed points. But it also characterizes attractive fixed points:

(4.10) Corollary. Let $X$ be a metric ANR, $x_{0} \in X, f: X \rightarrow X a$ map of compact attraction, $x_{0}$ an attractive fixed point of $f$ relative $U \in U\left(x_{0}\right)$ and $f(x) \neq x$ for all $x \in \partial U$. Assume there is $V \in U\left(x_{0}\right)$, $\operatorname{cl} V \subset U$, such that $j: \operatorname{cl} V \rightarrow X$ induces isomorphisms in $H_{*}$. Then

$$
i(X, f, U)=\Lambda(f, X)
$$

Proof. Set $W=X \backslash \mathrm{cl} V$; then $i: X \backslash W \rightarrow X$ induces isomorphisms in $H_{*}$. Since $x_{0}$ is attractive there is an $n_{0} \in \mathbf{N}$ such that $f^{n}(\operatorname{cl} V) \subset$ $f^{n}(U) \subset V$, i.e. $f^{n}(X \backslash W) \subset V=X \backslash$ cl $W$, whenever $n \geqq n_{0}$. 
(4.10) provides a characterization of attractive fixed points without the assumption that the fixed point has an acyclic neighbourhood (cf. [19], Theorem 3).

(4.11) Example. Let $D=\left\{x \in \mathbf{R}^{2} \mid\|x\| \leqq 1\right\}, U=\left\{x \in \mathbf{R}^{2} \mid\|x\|<\right.$ $1 / 2\}$ and $f: D \rightarrow D$ the following mapping:

Define

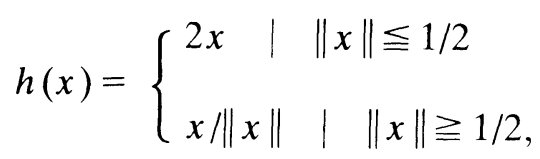

let $g: D \rightarrow D$ be an irrational rotation; and set $f=g \circ h$. Then 0 is the only fixed point of $f$ in $D$. Moreover, 0 is a repulsive fixed point relative to $U$ and clearly $i(D, f, U)=1$.

Note that assumption (4.8.2) is not satisfied here and that, in view of the existence of non-ejective fixed points (cf. Corollary (4.5.2)) 0 is the only fixed point of $f$ in $D$.

V. A remark on semi-flows. Lemma (4.3) suggests the following observation. If the assumptions are those of (4.3) and in addition $f$ is homotopic to $i d_{X}$ and $f_{I X \backslash F}$ is homotopic to $i d_{X \backslash F}$, then

$$
i(X, f, W)=\chi(X)-\chi(X \backslash F)
$$

where $\chi$ denotes the Euler characteristic.

(5.1) Definition. Let $X$ be a topological space and $\Phi=\left\{\phi_{t}\right\}_{t \in \mathbf{R}_{+}}$a continuous family of maps $\phi_{t}: X \rightarrow X$.

(5.1.1) $\Phi$ is said to be a semi-flow on $X$ provided $\phi_{t_{1}+t_{2}}=\phi_{t_{1}} \circ \phi_{t_{2}}$ and $\phi_{0}=i d_{x}$.

(5.1.2) A point $x_{0} \in X$ is said to be an ejective stationary point for $\Phi$ relative to $U \in U\left(x_{0}\right)$ provided $\phi_{t}\left(x_{0}\right)=x_{0}$ for all $t \geqq 0$; and for all $x \in U \backslash\left\{x_{0}\right\}$ there is a $t$ such that $\phi_{t}(x) \notin U$.

(5.2) Proposition. Let $\Phi=\left\{\phi_{t}\right\}_{t \in \mathbf{R}_{+}}$be a semi-flow on $X$. Then $\left\{\phi_{t}\right\}_{t \in \mathbf{Q}_{+}}$is divisible (i.e. for any pair $t_{1}, t_{2} \in \mathbf{Q}_{+}$there is $t_{0} \in \mathbf{Q}_{+}$such that for some $\left.n_{1}, n_{2} \in \mathbf{N} \phi_{t_{1}}=\left(\phi_{t_{1}}\right)^{n_{i}}, i=1,2\right)$. Assume that for any $t>0$ we have that $\operatorname{Fix}\left(\phi_{t}\right) \neq \varnothing$ and compact; then $\bigcap_{t} \operatorname{Fix}\left(\phi_{t}\right) \neq \varnothing$ and compact.

(5.3) Proposition. Let $X$ be a metric ANR, $\Phi$ a semi-flow on $X$, 
$\phi_{t}: X \rightarrow X$ a map of compact attraction for all $t>0$ and $x_{0} \in X$ such that $x_{0}$ is an ejective stationary point of $\Phi$ relative to $U \in \mathcal{U}\left(x_{0}\right)$ and $\phi_{t}(x) \neq x$ for all $x \in \partial U$ and $t>0$. Then, $\chi(X)$ and $\chi\left(X \backslash\left\{x_{0}\right\}\right)$ are defined and

$$
i(X, \Phi, U)=\chi(X)-\chi\left(X \backslash\left\{x_{0}\right\}\right)
$$

and

$$
i(X, \Phi, X \backslash \mathrm{cl} U)=\chi\left(X \backslash\left\{x_{0}\right\}\right)
$$

where $i(X, \Phi, U)=i\left(X, \phi_{t}, U\right), t>0$.

Proof. Fix $t>0$. Then $x_{0}$ is an ejective fixed point of $\phi_{t}$ relative to $U \in U\left(x_{0}\right)$. Applying Corollary (4.4) we obtain

$$
i\left(X, \phi_{t}, U\right)=\Lambda\left(\phi_{t}, X\right)-\Lambda\left(\phi_{t|X|\{x 0\}}, X \backslash\left\{x_{0}\right\}\right) .
$$

Now $\left(\phi_{t}\right)_{*}=\left(i d_{X}\right)_{*}$ and $\left(\phi_{t|X|\left\{x_{0}\right\}}\right)_{*}=\left(i d_{X \mid\left\{x_{0}\right\}}\right)_{*}$ are Leray endomorphisms, thus, $H_{*}(X)$ and $H_{*}\left(X \backslash\left\{x_{0}\right\}\right)$ are of finite type and the conclusion follows from the definition of the generalized Lefschetz number.

(5.4) EXAMPLE.

(5.4.1) If $X=S^{2}$ (the two dimensional sphere), $x_{0} \in X$ is an ejective stationary point of a semi-flow $\Phi$ on $S^{2}$, then

$$
i\left(S^{2}, \Phi, S^{2} \backslash \mathrm{cl} U\right)=\chi\left(S^{2} \backslash\{\text { point }\}\right)=1
$$

and Proposition (5.2) implies that $\Phi$ must have another stationary point.

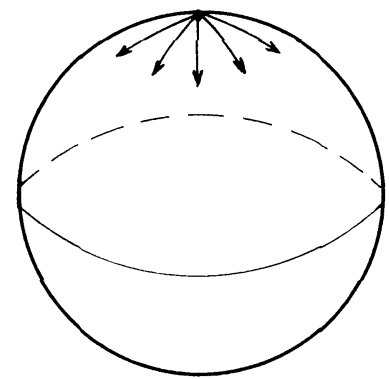

FiguRE 1

(5.4.2) Let $K$ be a cross in $\mathbf{R}^{2}$ centered at 0 . Let $\Phi$ be a semi-flow on $K$ such that 0 is an ejective stationary point of $\Phi$ relative to $U$. Then

$$
\begin{gathered}
i(K, \Phi, U)=\chi(K)-\chi(K \backslash\{0\})=1-4=-3 \text { and } \\
i(K, \Phi, K \backslash \operatorname{cl} U)=4,
\end{gathered}
$$


which suggests that the four "endpoints" of $K$ have to be stationary points.

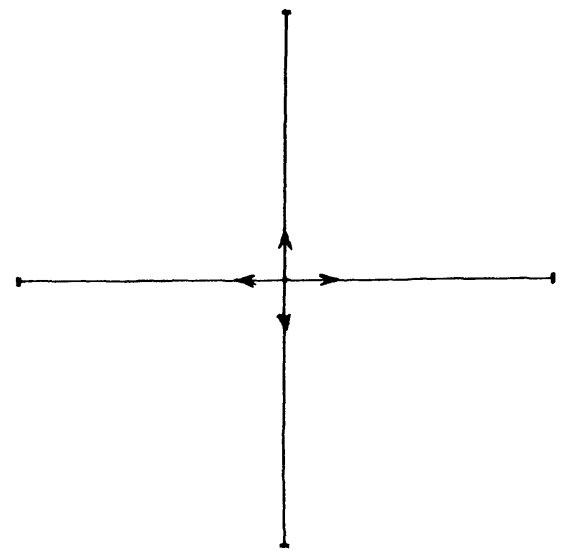

FiguRE 2

\section{REFERENCES}

1. C. Bowszyc, Fixed point theorems for the pairs of spaces, Bull. Acad. Polon. Sci., Ser. Math. Astronom. Phys. 16 (1968), 845-850.

2. F. E. Browder, Another generalization of the Schauder fixed point theorem, Duke Math. J., 32 (1965), 399-406.

3. - A further generalization of the Schauder fixed point theorem, Duke Math. J., 32 (1965), $575-578$.

4. - Asymptotic fixed point theorems, Math. Ann., 185 (1970), 38-60.

5. R. F. Brown, Notes on Leray's index theory, Advances in Math., 7 (1971), 1-28.

6. C. C. Fenske, H. O. Peitgen, Repulsive fixed points of multivalued transformations and the fixed point index, Math. Ann., 218 (1975), 9-18.

7. G. Fournier, Généralisations du théorème de Lefschetz pour des espaces non-compacts I, II, III, Bull. Acad. Polon. Sci., Ser. Math. Astronom. Phys., 23 (1975), 693-711.

8. A. Granas, The Leray-Schauder index and the fixed point theory for arbitrary ANR's, Bull. Soc. math. France, 100 (1972), 209-228.

9. V. Klee, Some topological properties of convex sets, Trans. Amer. Math. Soc., 78 (1955), 30-45.

10. J. Leray, Théorie des points fixes: indice total et nombre de Lefschetz, Bull. Soc. math. France, 87 (1959), 221-233.

11. Sur la position d' un ensemble fermé de points d'un espace topologique, J. Math. Pures Appl., 24 (1945), 169-199.

12. R. D. Nussbaum, Asymptotic fixed point theorems, Math. Ann., 185 (1970), 38-60.

13. - Some asymptotic fixed point theorems, Trans. Amer. Math. Soc., 171 (1972), 349-375.

14. - Periodic solutions of some nonlinear, autonomous functional differential equations II, J. Differential Equations, 14 (1973), 360-394.

15. - Periodic solutions of some nonlinear, autonomous functional differential equations, Ann. Mat. Pura Appl., 101 (1974), 263-306.

16. - A global bifurcation theorem with applications to functional differential equations, $J$. Functional Analysis, 19 (1975), 319-338.

17. H. O. Peitgen, Asymptotic fixed point theorems and stability, J. Math. Anal. Appl., 47 (1974), $32-42$. 
18. - On the Lefschetz number for iterates of continuous mappings, Proc. Amer. Math. Soc., 54 (1976), 441-444.

19. - Some applications of the fixed point index in asymptotic fixed point theory, Proc. Conf. on Fixed Point Theory and its Applications, Halifax 1975, ed.: Academic Press N.Y. 1976.

20. H. Steinlein, Ein Satz über den Leray-Schauderschen Abbildungsgrad, Math. Z., 120 (1972), 176-208.

21. , Über die verallgemeinerten Fixpunktindizes von Iterierten verdichtender Abbildungen, manuscripta math., 8 (1972), 251-266.

22. — A new proof of the $(\bmod p)$-theorem in asymptotic fixed point theory, Proc. Conf. on Problems in Nonlinear Functional Analysis, Bonn 1974, ed.: Ber. Ges. Math. Datenverarb. Bonn, 103 (1975), 29-42.

23. P. P. Zabreǐko, M. A. Krasnosel'skiǐ, Iterations of operators and the fixed point index, Doklady Akad. Nauk SSSR 196 (1971), 1006-1009. = Soviet Math. Dokl., 12 (1971), 294-298.

Received January 7, 1976. This work was supported by the Deutsche Forschungsgemeinschaft, Sonderforschungsbereich 72 an der Universität Bonn

Mathematisches INSTITUT

DER JUSTUS LIEBIG-UNIVERSITÄT

D-6300 Giessen, Arndtstrasse 2

AND

INSTITUT FÜR ANGEWANDTE MathEMATIK

DER RHEINISCHEN FRIEDRICH-WILHELMS-UNIVERSITÄT

D-5300 Bonn, Wegelerstrasse 6 


\section{PACIFIC JOURNAL OF MATHEMATICS}

\section{EDITORS}

RICHARI) ARENS (Managing Editor)

University of California

Los Angeles, CA 90024

R. A. Beaumiont

University of Washington

Seattle, WA 98105

\section{J. DugunduI}

Department of Mathematics University of Southern California Los Angeles, CA 90007

D. Gilbarg and J. Milgram Stanford University

Stanford, CA 94305

\section{ASSOCIATE EDITORS}

E. F. BECKENBACH
B. H. NEUMANN

F. Wolf

K. YoshidA

\section{SUPPORTING INSTITUTIONS}

UNIVERSITY OF BRITISH COLUMBIA CALIFORNIA INSTITUTE OF TECHNOLOGY

UNIVERSITY OF CALIFORNIA

MONTANA STATE UNIVERSITY

UNIVERSITY OF NEVADA

NEW MEXICO STATE UNIVERSITY

OREGON STATE UNIVERSITY

UNIVERSITY OF OREGON

OSAKA UNIVERSITY

\author{
UNIVERSITY OF SOUTHERN CALIFORNIA \\ STANFORD UNIVERSITY \\ UNIVERSITY OF HAWAII \\ UNIVERSITY OF TOKYO \\ UNIVERSITY OF UTAH \\ WASHINGTON STATE UNIVERSITY \\ UNIVERSITY OF WASHINGTON \\ AMERICAN MATHEMATICAL SOCIETY
}

The Supporting Institutions listed above contribute to the cost of publication of this Journal, but they are not owners or publishers and have no responsibility for its contents or policies.

Mathematical papers intended for publication in the Pacific Journal of Mathematics should be in typed form or offset-reproduced (not dittoed), double spaced with large margins. Underline Greek letters in red, German in green, and script in blue. The first $p: 1<$ graph or two must be capable of being used separately as a synopsis of the entire paper. Items of the biblography should not be cited there unless absolutely necessary, in which case they must he identified by author and Journal, rather than by item number. Manuscripts, in duplicate, may be sent to any one of the four editors. Please classify according to the scheme of Math. Reviews, Index to Vol. 39. All other communications should be addressed to the managing editor, or Elaine Barth, University of California, Los Angeles, California, 90024.

100 reprints are provided free for each article, only if page charges have been substantially paid. Additional copies may be obtained at cost in multiples of 50 .

The Pacific Journal of Mathematics is issued monthly as of January 1966. Regular subscription rate: $\$ 72.00$ a year (6 Vols., 12 issues). Special rate: $\$ 36.00$ a year to individual members of supporting institutions.

Subscriptions, orders for back numbers, and changes of address should be sent to Pacific Journal of Mathematics, 103 Highland Boulevard, Berkeley, California, 94708.

PUBLISHED BY PACIFIC JOURNAL OF MATHEMATICS, A NON-PROFIT CORPORATION Printed at Jerusalem Academic Press, POB 2390, Jerusalem, Israel.

\section{Copyright (C) 1976 Pacific Journal of Mathematics} All Rights Reserved 


\section{Pacific Journal of Mathematics}

\section{Vol. 66, No. 2 December, 1976}

Gerald A. Beer, Tax structures whose progressivity is inflation neutral..... 305

William M. Cornette, A generalization of the unit interval............. 313

David E. Evans, Unbounded completely positive linear maps on

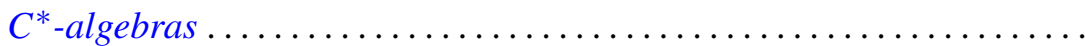

Hector O. Fattorini, Some remarks on convolution equations for

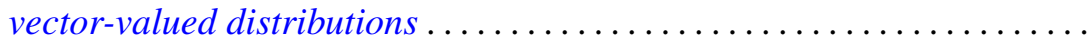

Amassa Courtney Fauntleroy, Automorphism groups of unipotent groups of

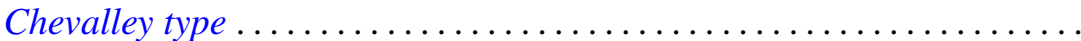

Christian C. Fenske and Heinz-Otto Peitgen, On fixed points of zero index in

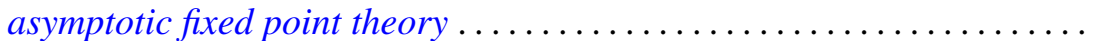

Atsushi Inoue, On a class of unbounded operator algebras. II ............

Herbert Meyer Kamowitz, The spectra of endomorphisms of algebras of

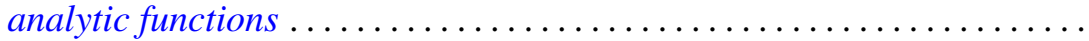

Jimmie Don Lawson, Embeddings of compact convex sets and locally compact cones ....................................

William Lindgren and Peter Joseph Nyikos, Spaces with bases satisfying certain order and intersection properties .....................

Emily Mann Peck, Lattice projections on continuous function spaces ...... 477

Morris Marden and Peter A. McCoy, Level sets of polynomials in $n$ real variables...

Francis Joseph Narcowich, An imbedding theorem for indeterminate Hermitian moment sequences......................

John Dacey O'Neill, Rings whose additive subgroups are subrings ...

Chull Park and David Lee Skoug, Wiener integrals over the sets bounded by sectionally continuous barriers .....................

Vladimir Scheffer, Partial regularity of solutions to the Navier-Stokes equations.

Eugene Spiegel and Allan Trojan, On semi-simple group algebras. II

Katsuo Takano, On Cameron and Storvick's operator valued function space integral 